EPJ Web of Conferences 5, 05005 (2010)

DOI:10.1051/epjconf/20100505005

C Owned by the authors, published by EDP Sciences, 2010

\title{
Astronomical polarimetry in the new era - applications and challenges
}

M. Redfern

Centre for Astronomy, National University of Ireland Galway, Galway, Ireland 